OPEN ACCESS

*Environmental Science Center, Qatar University, P.O. Box 2713, State of Qatar

Corresponding author email address: jeff.obbard@qu.edu.qa 10.5339/qproc.2015.qulss2015.35

Copyright: 2015 Azenith Castillo, Jeffrey Philip Obbard,

licensee Bloomsbury Qatar Foundation Journals. This is an open access article distributed under the terms of the Creative Commons Attribution license CC BY 4.0, which permits unrestricted use, distribution and reproduction in any medium, provided the original work is properly cited.
Qatar University Life Science Symposium-QULSS 2015 Global Changes: The Arabian Gulf Ecosystem

\section{Prevalence of microplastics in the marine environment of Qatar's exclusive economic zone}

\author{
Azenith Castillo*, Jeffrey Philip Obbard
}

Microplastics are now firmly recognized as a ubiquitous and growing global threat to marine biota, as well as their associated ecology and habitats. The prevalence of microplastics in the marine waters within Qatar's Exclusive Economic Zone (EEZ) was investigated. Following sample extraction, microplastics were characterized using Attenuated Total Reflectance-Fourier Transform Infrared (ATR-FTIR) spectroscopy, and the polymer type then verified using OMNIC Spectra Library. Synthetic polymer microplastics were identified in seawater sampled from ten out of twelve marine monitoring stations, with varying shapes and sizes. Granular shaped microplastics present ranged from $120 \mu \mathrm{m}$ to $1.2 \mathrm{~mm}$ in size, and fibrous microplastic ranged from $150 \mu \mathrm{m}$ to 10mm. Polypropylene (PP), low density polyethylene (LDPE), polyethylene (PE), polystyrene (PS), polyamide (PA), acrylonitrile butadiene styrene (ABS), polymethyl methacrylate (PMMA) and cellophane microplastic particles were identified. Copolymers were also identified from the collected samples, including poly(styrene:vinylidene chloride), poly(ethylene:propylene:diene) and alkyd resin e.g. paint debris. The most numerous type of synthetic polymer microplastic identified was polypropylene, with the greatest concentration found near Halul Island in the north east of the Qatar EEZ, where nearby anthropogenic activities include oil-rig installations and shipping operations. In addition, as par to the investigation, an optimized method was also developed for the extraction of microplastics from seawater samples rich in phytoplankton. The use of $1 \mathrm{M} \mathrm{NaOH}$ proved to be more efficient digestion treatment than $10 \mathrm{M} \mathrm{NaOH}$ and 22.5M HNO3 solutions, as the latter two solutions resulted in structural damage and discoloration of the reference polymers: polyvinyl alcohol (PVA), polyvinyl chloride (PVC), polystyrene (PS), polyethylene (PE), and nylon. 